\title{
DALM, rest in peace: a pathologist's perspective on dysplasia in inflammatory bowel disease in the post-DALM era
}

\author{
Kenrry Chiu ${ }^{1}$ Robert H. Riddell ${ }^{2}$ - David F. Schaeffer ${ }^{1}$
}

Received: 23 January 2018 / Revised: 30 March 2018 / Accepted: 1 April 2018 / Published online: 22 May 2018

(c) United States \& Canadian Academy of Pathology 2018

\begin{abstract}
There are few abbreviations in surgical pathology that are associated with as much immediate recognition, frustration, and confusion as DALM (dysplasia-associated lesion or mass). DALM is used to describe endoscopically visible dysplastic lesions in the surveillance of patients with inflammatory bowel disease. However, the diagnosis of DALM has been complicated by the inconsistent criteria and use of terminology for describing dysplasia in inflammatory bowel disease, and a tendency to relate DALM with the need for colectomy. Fortunately, advancements in both endoscopic visualization and local excision capability have allowed for a more defined management of dysplasia in inflammatory bowel disease. In 2015, the Surveillance for Colorectal Endoscopic Neoplasia Detection and Management in Inflammatory Bowel Disease Patients International Consensus Recommendations (SCENIC) Development Panel, a panel of predominantly expert gastroenterologists and endoscopists in surveillance of inflammatory bowel disease, published a consensus statement. One recommendation was to abandon DALM-related terminology in favor of endoscopic descriptors modified from the Paris endoscopic classification. Recommendations on surveillance and management of dysplastic lesions were also provided. Nevertheless, interval carcinomas and metachronous neoplasia remain persistent issues. This review aims to provide an update on the post-DALM terminology and management recommendations for inflammatory bowel disease-associated dysplasia necessary for a meaningful communication between pathologists and clinicians.
\end{abstract}

\section{Introduction}

Patients with inflammatory bowel disease have an increased life-time risk of colorectal cancer [1]. Although the first case report of cancer complicating ulcerative colitis is frequently attributed to Crohn and Rosenberg in 1925 [2], it was actually described almost two decades earlier by $\mathrm{P}$. Lockhart-Mummery from St. Mark's Hospital in London, following the purchase of his new electric sigmoidoscope [3]. Patients with inflammatory bowel disease are monitored regularly by surveillance colonoscopies to detect dysplasia $[4,5]$, the precursor of colorectal cancer, and sometimes "early" carcinoma. Dysplasia can be endoscopically

David F. Schaeffer

david.schaeffer@vch.ca

1 Division of Anatomic Pathology, Vancouver General Hospital, University of British Columbia, Vancouver, BC, Canada

2 Department of Pathology and Laboratory Medicine, Mount Sinai Hospital, University of Toronto, Toronto, ON, Canada invisible or visible [6]. In 1981, dysplasia-associated lesion or mass (DALM) was coined to describe visible dysplastic lesions identified on colonoscopy in patients with inflammatory bowel disease [7]. Historically, DALMs were often associated with invasive carcinoma [7, 8], for which surgical resection was recommended. The diagnosis of DALM has therefore been a source of consternation for the surgical pathologist. Contributing to the apprehension was the difficulty in distinguishing inflammatory bowel diseaseassociated polypoid dysplasia and sporadic adenomas occurring within colitic mucosa [9], the former potentially leading to colectomy while the latter would be followed up conservatively after endoscopic removal.

However, it has been shown that not all visible dysplastic lesions, i.e., DALMs, require colectomy [10-12]. Nevertheless, the perceived gravity of the diagnosis of a DALM persists. In light of this issue and other advancements in endoscopic visualization and local endoscopic excision of dysplastic lesions, the Surveillance for Colorectal Endoscopic Neoplasia Detection and Management in Inflammatory Bowel Disease Patients International Consensus Recommendations (SCENIC) Development Panel published 
a consensus statement in 2015 [6]. This international panel, composed primarily of expert gastroenterologists and therapeutic endoscopists in inflammatory bowel disease surveillance, recommended that DALM-related terminology be abandoned. In its place, the panel recommended that standardized terminology for describing the endoscopic appearance of dysplastic lesions, adapted from the Paris endoscopic classification, be used [6]. This recommendation has been adopted by the American Society for Gastrointestinal Endoscopy [5] and the European Crohn's and Colitis Organization [13]. The panel also recommended chromoendoscopy as the preferred modality for surveillance colonoscopies. Following complete endoscopic removal of endoscopically visible dysplastic lesions, continuation of surveillance rather than colectomy was recommended. The aim of this review is to provide an update on the terminology, management recommendations, and controversies with regard to dysplasia in inflammatory bowel disease, in order to facilitate meaningful communication between pathologists and clinicians.

\section{From DALM to the SCENIC view}

\section{Dysplasia-associated lesions or mass}

Historically, DALM was a helpful concept that emphasized the high risk of cancer associated with a visible dysplastic lesion. In its broadest definition, a DALM was an endoscopically visible, elevated, or polypoid lesion found in the context of inflammatory bowel disease that showed only dysplasia without associated invasive carcinoma on microscopic examination. This was in contrast to endoscopically invisible dysplasia, often referred to as flat dysplasia [14, 15]. DALM was coined by Blackstone et al. in 1981 in their study of 112 patients with ulcerative colitis [7]. They reported that of 12 patients who presented with DALM, 7 (58\%) were found to have invasive carcinoma in resection specimens despite the biopsies showing only low-grade dysplasia in 5 patients and high-grade dysplasia in the other 2. They concluded that a DALM was an indication for colectomy due to the high likelihood of associated cancer, even if the biopsy did not indicate carcinoma. This recommendation was also advocated by others in the 1980 s and 1990s [8, 16, 17]. It was understood that a biopsy of a DALM may not reveal underlying invasive carcinoma, and that colectomy would be required to exclude carcinoma [7, 18].

The concept of a DALM as a high-risk marker for malignancy resulted in difficulty when dealing with polypoid lesions in colitic mucosa. It was previously thought that the distinction between inflammatory bowel diseaseassociated dysplastic polypoid lesion (i.e., caused by inflammatory bowel disease) and a sporadic adenoma (presumably sporadic and unrelated to inflammatory bowel disease) was important; the former presumably required colectomy and the latter was treated by endoscopic polypectomy, a much less aggressive treatment [19]. However, inflammatory bowel disease-associated polypoid dysplasia was often endoscopically indistinguishable from sporadic adenomas [9, 19]. On histology, features like dysplasia in the stalk of the polyp or adjacent mucosa, when present, was said to indicate inflammatory bowel disease-associated dysplasia [18]. Younger age, chronic lamina propria inflammation, and a mixture of benign and dysplastic crypts at the surface were described to favor inflammatory bowel disease-associated dysplasia over a sporadic adenoma [9, 19]. Nevertheless, the distinction between inflammatory bowel disease-associated polypoid dysplasia and sporadic adenomas based on histologic criteria remains subjective and can be difficult or impossible in day-to-day practice $[9$, 18-20].

In the 1990s, two groups showed that polypoid dysplasia in colitic mucosa without associated flat dysplasia could be removed by endoscopic polypectomy and followed with surveillance, thereby avoiding the need for colectomy [10-12]. Rubin et al. reported no carcinoma or flat dysplasia amongst 48 such patients after a mean follow-up period of 4.1 years [12]. Another group based in Boston looked at 24 patients with adenoma-like DALM who, after a mean follow-up period of 82.1 months, showed one patient with carcinoma and one with flat dysplasia on colectomy $[10,11]$. The authors concluded that the rate of carcinoma and flat dysplasia was acceptably low following endoscopic resection of polypoid lesions without associated flat dysplasia. This was further supported by a meta-analysis that reported a low risk of colorectal cancer following resection of polypoid dysplasia [21]. Therefore, determining if an endoscopically resectable polypoid dysplastic lesion was an inflammatory bowel disease-associated lesion or a sporadic adenoma was no longer necessary from a therapeutic standpoint; endoscopic polypectomy, with biopsies around the base of the polyp to ensure that local excision was complete, sufficed either way.

However, the diagnostic issue of inflammatory bowel disease-associated dysplasia vs. sporadic adenomas for polypoid lesions led to inconsistency in the use of the DALM terminology. The term "adenoma-like" DALMs may be used to describe polypoid dysplasia within colitic mucosa regardless of etiology [19]. Others, however, differentiated between sporadic adenomas and DALMs, the latter presumed to be inflammatory bowel diseaseassociated [14]. Even "flat" dysplasia was inconsistently defined, as some authors used the term to describe visible, slightly elevated lesions rather than just endoscopically invisible dysplasia found on random biopsy $[15,22]$. The 
lack of uniformity in terminology for dysplasia led to the suggestion that terminology be standardized to eliminate confusion [23, 24].

\section{Chromoendoscopy and current endoscopy}

Also contributing to the changing landscape of dysplasia in inflammatory bowel disease were improvements in endoscopic visualization. It was previously believed that most cases of dysplasia were endoscopically invisible [15], but it is now well recognized that the majority of dysplasia is identified on targeted biopsies of visible lesions [14, 25, 26]. Furthermore, there is speculation that dysplasia that would have been endoscopically invisible in the past would be visible with newer endoscopic techniques [27, 28]. Indeed, increased detection rates of visible dysplastic lesions have been demonstrated for high-definition endoscopy compared to standard-definition endoscopy [29]. Furthermore, chromoendoscopy, which is the application of dye on colonic mucosa to enhance the contrast of epithelial surfaces [6], has been shown to detect additional dysplastic lesions in comparison to white-light endoscopy [30-39], especially flat, non-polypoid lesions [24]. More recently, full-spectrum endoscopy, which increases the field of view compared to conventional forward viewing endoscopy, was shown to increase the number of detected dysplastic lesions in the setting of surveillance in inflammatory bowel disease [40].

\section{The SCENIC consensus statement: endoscopic descriptors of lesions and visualization}

The SCENIC Guideline Development Panel published a consensus statement in 2015 in regard to endoscopic techniques for surveillance colonoscopy and the management of dysplasia in inflammatory bowel disease. As part of an updated framework, the panel advised that the DALM terminology be abandoned in favor of standardized definitions that focus on the resectability and the endoscopic appearance of lesions with the aim to establish uniformity in communication [6].

A lesion is endoscopically resectable if: "(1) distinct margins of the lesion could be identified, (2) the lesion appears to be completely removed on visual inspection after endoscopic resection, (3) histologic examination of the resected specimen is consistent with complete removal, and (4) biopsy specimens taken from mucosa immediately adjacent to the resection site are free of dysplasia on histologic examination" [6]. The application of these criteria for pathologists is discussed in the next section.

The endoscopic appearance of lesions are described using terminology adapted from the Paris endoscopic classification [41] (Table 1). Importantly, invisible dysplasia is dysplasia identified on random, non-targeted biopsies of mucosa without a visible lesion; visible dysplasia is dysplasia identified on biopsies from a lesion visualized at colonoscopy. Visible dysplasia can be polypoid or non-polypoid (Fig. 1). Polypoid lesions are raised and are pedunculated or sessile. Nonpolypoid lesions can be superficial elevated, flat, or depressed.

\section{Clinical management of specimens from surveillance colonoscopies}

The SCENIC consensus statement made several recommendations on surveillance colonoscopy and follow-up of dysplasia in inflammatory bowel disease. Chromoendoscopy instead of white-light endoscopy was recommended based on data demonstrating increased detection of dysplastic lesions in the former [6], including data from randomized clinical trials [36, 37]. High-definition endoscopy instead of standard-definition endoscopy was also recommended. Surveillance following the complete endoscopic removal of visible dysplastic lesions was also emphasized. However, these recommendations and suggestions were
Table 1 Terminology for the endoscopic appearance of lesions in surveillance for dysplasia in inflammatory bowel disease adopted by the SCENIC Guideline Development Panel

\begin{tabular}{cl}
\hline Term & Definition \\
\hline Visible dysplasia & $\begin{array}{l}\text { Dysplasia identified on targeted biopsies from a lesion visualized at } \\
\text { colonoscopy } \\
\text { Polypoid }\end{array}$ \\
Pedunculated & Lesion protruding from the mucosa into the lumen $\geq 2.5 \mathrm{~mm}$ \\
Sessile & Lesion not attached to the mucosa by a stalk \\
Non-polypoid & Lesion with little $(<2.5 \mathrm{~mm})$ or no protrusion above the mucosa \\
Superficial elevated & Lesion with protrusion but $<2.5 \mathrm{~mm}$ above the lumen \\
Flat & Lesion without protrusion above the mucosa \\
Depressed & Lesion with at least a portion depressed below the level of the mucosa \\
Invisible dysplasia & Dysplasia identified on random (non-targeted) biopsies of colon mucosa \\
& without a visible lesion
\end{tabular}

Source: Laine et al. [6] 

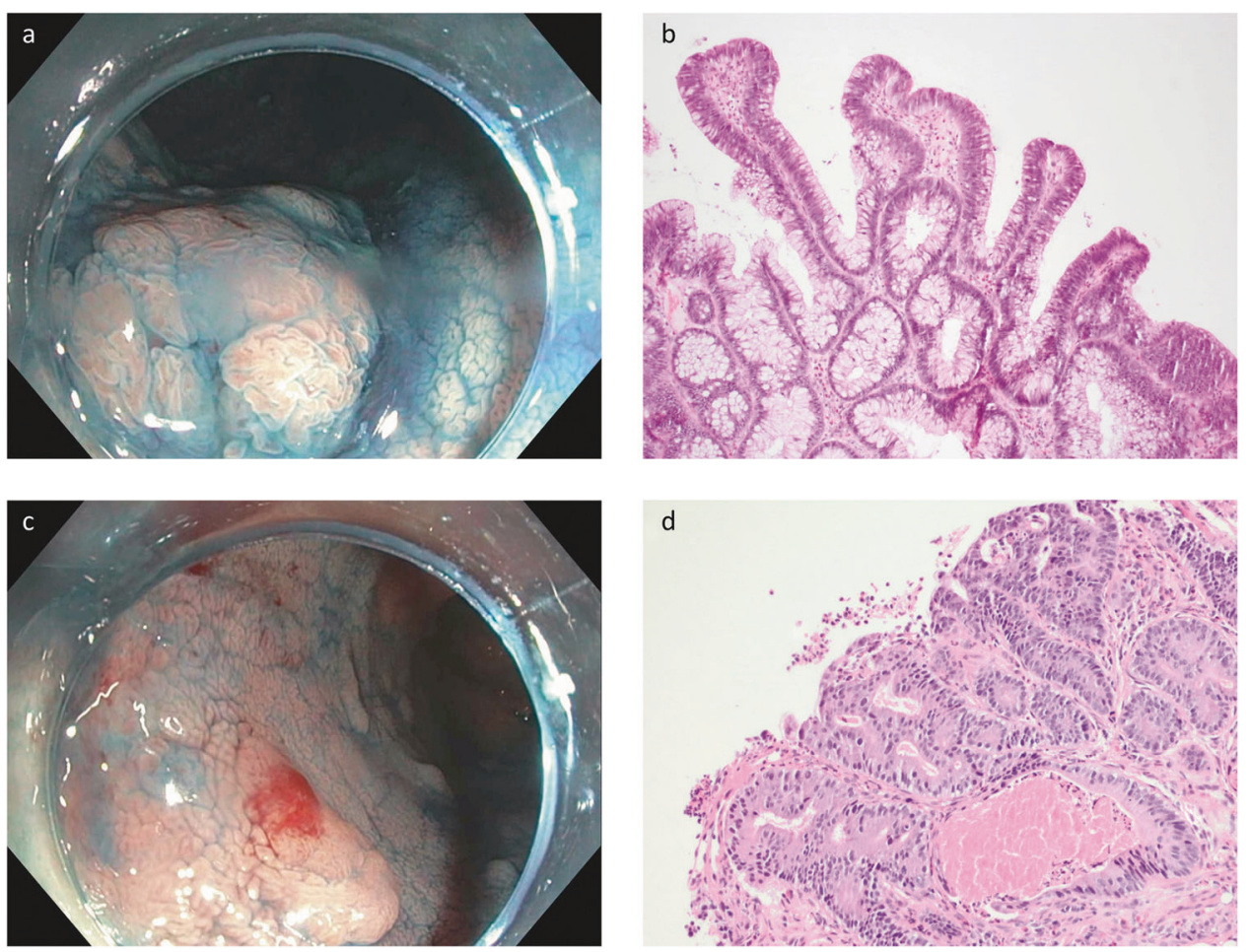

Fig. 1 Chromoendoscopy and histology of endoscopically visible lesions in ulcerative colitis. a Polypoid sessile lesion seen on chromoendoscopy. The borders of the polyp were accentuated by the blue dye. The endoscopic mucosal resection of the polyp b showed polypoid low-grade dysplasia with crowded, elongated, and hyperchromatic nuclei. c Non-polypoid superficial elevated lesion seen on chromoendoscopy. The application of blue dye aids in the visualization of non-polypoid lesions. $\mathbf{d}$ The biopsy of a different case of a non-

polypoid elevated lesion showing high-grade dysplasia. The epithelium showed marked cytologic atypia, loss of polarity, and cribriform glandular architecture. Definitive evidence of invasive carcinoma was not identified. As the lesion was not endoscopically resectable, the lesion was surgically resected. Invasive adenocarcinoma was identified in the colectomy specimen. Figures (a) and (c) are courtesy of Dr. Fergal Donnellan, Division of Gastroenterology, Vancouver General Hospital, Vancouver, Canada

made by a group of predominantly therapeutic endoscopists and gastroenterologists based on limited data. The group identified no studies that compared surveillance colonoscopy to colectomy for neither endoscopically resectable visible dysplasia nor endoscopically invisible dysplasia. Recommendations and suggestions in regard to the management of dysplastic lesions, visible or invisible, were based on very low-quality evidence as judged by the group [6]. A panel with more surgeons and pathologists may have offered different conclusions from the ones presented by the SCENIC panel, particularly for nonpolypoid visible dysplasia and the role of random biopsies, as discussed below.

The major role of the pathologist in surveillance colonoscopies for inflammatory bowel disease is to identify dysplasia or features suggesting invasive carcinoma, bearing in mind that carcinomas in inflammatory bowel disease can arise directly from low-grade dysplasia (or less) [42]. Determination of whether a specimen is from a polypectomy, complete endoscopic resection (endoscopic mucosal resection or endoscopic submucosal dissection),

incomplete resection, biopsy of a visible lesion, or a random biopsy should be noted.

The pathologist should report on the absence or presence of dysplasia. When dysplasia is present, the grade of dysplasia should also be reported (Fig. 1). The major classification system in North America for reporting dysplasia consists of four categories as described by Riddell et al. [18] (Table 2): (1) negative for dysplasia, (2) indefinite for dysplasia, (3) low-grade dysplasia, and (4) high-grade dysplasia. The Vienna system [43], used more often in Japan and Europe, consists of the same classification but with additional categories (Table 2). Use of either system is appropriate, and depends on local or institutional preference. The reader is encouraged to consult gastrointestinal pathology textbooks [44-46] or other references [18, 43, 47-49] for a detailed discussion of the histologic features of dysplasia.

In this section, the approach to the pathologic reporting and subsequent management of the various types of lesions are described (Fig. 2). Controversies and issues regarding management are discussed. 
Table 2 The Riddell and Vienna Classification System for dysplasia in inflammatory bowel disease

\begin{tabular}{ll}
\hline Riddell & Vienna \\
\hline Negative for dysplasia & Negative for neoplasia/dysplasia \\
Indefinite for dysplasia & Indefinite for neoplasia/dysplasia \\
Low-grade dysplasia & Non-invasive low-grade neoplasia \\
& Low-grade adenoma/dysplasia \\
High-grade dysplasia & Non-invasive high-grade neoplasia \\
& i. High-grade adenoma/dysplasia \\
& ii. Non-invasive carcinoma (carcinoma \\
& in situ) \\
& iii. Suspicion of invasive carcinoma \\
& Invasive neoplasia \\
& i. Intramucosal adenocarcinoma \\
& ii. Submucosal invasive carcinoma or \\
& beyond \\
\hline
\end{tabular}

Source: Riddell et al. [18] and Schlemper et al. [43]

\section{Endoscopically resectable visible dysplastic lesions}

In the SCENIC consensus statement, one criterion for confirmation of the endoscopic resectability of a dysplastic lesion is histologic examination that is "consistent with complete removal" [6]. However, this condition is often impossible to confirm on histologic examination if a lesion is resected piecemeal. In such cases, a comment indicating that histologic assessment of the completeness of excision is not possible due to piecemeal resection may be included.

As another criterion for the confirmation of endoscopic resectability, the SCENIC consensus statement suggested that biopsies of mucosa immediately adjacent to the resection site be taken and shown to be free of dysplasia on microscopic examination by the pathologist. This recommendation is endorsed in guidelines published by major societies [5, 6, 13]. The presence of dysplasia in these biopsies implies that the lesion has not been completely removed. However, the protocol of obtaining biopsies adjacent to a lesion (e.g., where to biopsy and how many biopsies) has not been standardized and this practice has not been well characterized in regard to patient outcomes [50]. The utility of this practice was recently assessed by a Dutch group on the speculation that improvement in the endoscopic visualization of lesions may have rendered this practice less useful [50]. In its study, dysplasia was found in biopsies of mucosa adjacent to dysplastic lesions in $5(3.7 \%)$ out of 136 lesions with low-grade dysplasia and 2 (50\%) of 4 lesions with highgrade dysplasia [50]. The rate of dysplasia in the biopsies adjacent to lesions was low overall, but the high rate of dysplasia in biopsies of mucosa adjacent to high-grade dysplasia, albeit based on a small sample, suggests that the borders of more advanced neoplasia may be more difficult to delineate and more prone to being incompletely resected. Therefore, biopsying around the base of resected lesions, particularly those with less welldelineated borders, may still be worthwhile.

\section{Polypoid lesions}

In the past, polypectomy specimens from colitic mucosa were a source of difficulty as distinguishing a sporadic adenoma from inflammatory bowel disease-associated polypoid dysplasia had significant therapeutic implications, with the latter potentially prompting colectomy. Not helping was a lack of reliable histologic criteria for making this distinction. Fortunately, the current view is that demonstrably complete polypectomy followed by continued surveillance is sufficient treatment for polypoid lesions in inflammatory bowel disease [5, 6, 13]. This approach was endorsed by the SCENIC Development Panel as a strong recommendation [6] based on data showing acceptably low rates of carcinoma with this practice [11,51-55], although the quality of the data was deemed as very low. A meta-analysis by Wanders et al. [21] also supports the view that the rate of colorectal cancer is low following the complete endoscopic resection of polypoid dysplasia.

The term DALM should be replaced by a descriptor like "polypoid low-grade dysplasia" as described in the SCENIC recommendations [6] based on the Paris endoscopic classification [41]. The descriptor "polypoid" avoids the historical connotation engendered by the term "DALM" of a lesion highly associated with malignancy. If there is concern from the clinician on whether the polypoid lesion is inflammatory bowel disease-associated, then a comment in the pathology report stating the following may be helpful: (1) the histologic distinction between sporadic adenoma and inflammatory bowel disease-associated polypoid dysplasia is unreliable, and (2) continued surveillance may be appropriate if complete endoscopic resection of the polypoid lesion was achieved.

If the lesion looks like a typical adenoma, then stating that the features suggest a sporadic adenoma can be appropriate, particularly when the lesion is present in noncolitic mucosa, i.e., mucosa not known to have been affected by chronic colitis. Signing out such a case as one would with an adenoma in a patient from the general population without inflammatory bowel disease may suffice.

\section{Non-polypoid lesions}

After complete removal of endoscopically resectable nonpolypoid dysplastic lesions, surveillance colonoscopy 
Fig. 2 Algorithm for management based on endoscopic and pathologic findings for inflammatory bowel disease patients undergoing surveillance colonoscopies. This is illustrated for a endoscopically resectable visible lesions, b endoscopically unresectable lesions, and c random biopsies of mucosa with no visible lesion. See text for details. "Findings that suggest incomplete resection include a biopsy of mucosa adjacent to the lesion showing dysplasia, or if a specimen is received intact, a margin positive for dysplasia

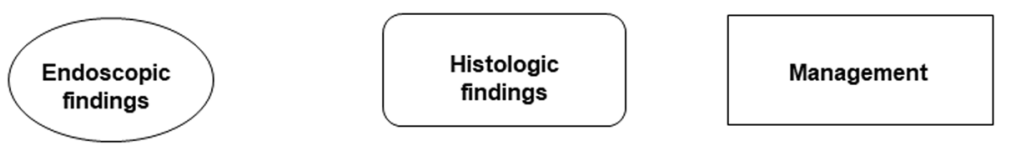

(a)

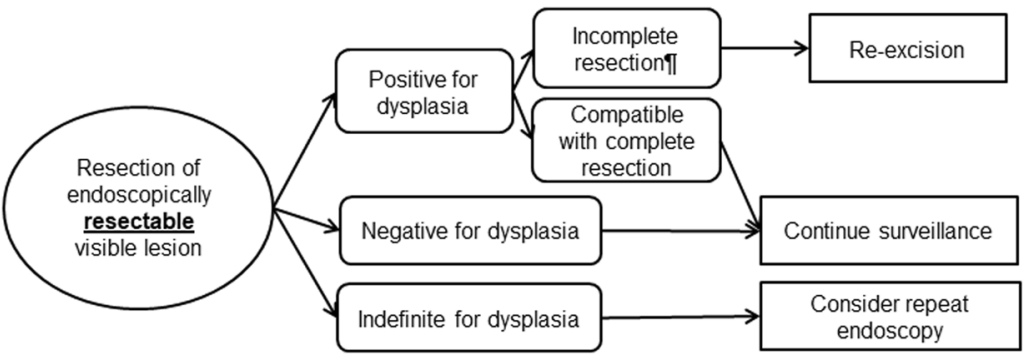

(b)

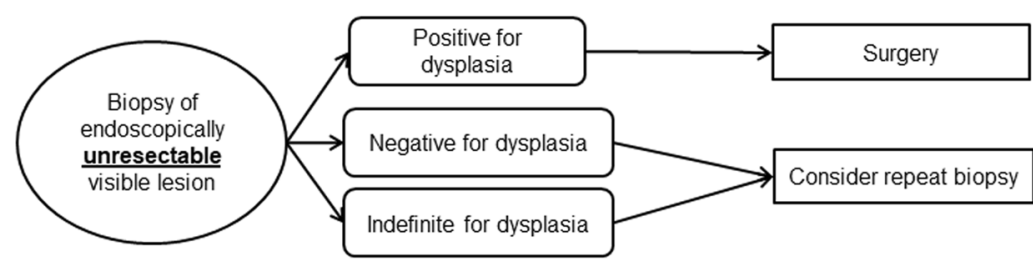

(c)



instead of colectomy was suggested by the SCENIC Development Panel [6], a recommendation given conditional strength. This recommendation was endorsed by the American Society for Gastrointestinal Endoscopy and the European Crohn's and Colitis Organization [5, 13]. This suggestion was similar to the recommendation for polypoid dysplasia, but in comparison, there is a paucity of data on outcomes following endoscopic resection of non-polypoid lesions. The SCENIC panel acknowledged the concern that non-polypoid lesions may be difficult to confidently resect completely, which is especially concerning since nonpolypoid lesions may be more high risk for colorectal cancer [6]. Borders of non-polypoid lesions can be difficult to delineate and removal of these lesions is technically challenging [56].

This recommendation diverged from previous guidelines favoring colectomy for non-polypoid dysplasia, as they were based on the premise that these lesions were endoscopically unresectable and associated with a high rate of synchronous carcinoma [7, 8, 22, 49].

\section{Endoscopically unresectable visible non-polypoid lesions}

If dysplasia is present on a biopsy of an endoscopically unresectable lesion, a colectomy is recommended due to the risk of associated invasive carcinoma [5, 13], since the biopsy may not sample the underlying invasive carcinoma [18]. A comment that an underlying, unsampled carcinoma cannot be excluded may be helpful.

\section{Random, non-targeted biopsy with invisible dysplasia}

Most dysplasia is visible [14]. A random biopsy with dysplasia implies endoscopically invisible dysplasia. Considering the potential consequence of colectomy (discussed subsequently), confirmation of the diagnosis of dysplasia with a gastrointestinal pathologist can be considered as suggested in the SCENIC consensus statement [6]. The basis for this suggestion is that inter-observer agreement for 
dysplasia in inflammatory bowel disease is poor to fair, with agreement on low-grade dysplasia being particularly problematic [57-59]. The SCENIC consensus statement also recommends a referral to an endoscopist with expertise in chromoendoscopy to determine if the dysplasia can be visualized [6], a recommendation given conditional strength. The rationale is that if a visible lesion is identified on chromoendoscopy in the vicinity of the random biopsy showing dysplasia, this lesion can then be managed as visible dysplasia as described above. If chromoendoscopy is not available or if no visible lesion is seen on chromoendoscopy, management is dependent on the grade of the dysplasia.

With increased detection of lesions with chromoendoscopy [36, 37], the role of random biopsies is controversial. The SCENIC panel did not reach a consensus on the role of random biopsies. In its pooled analysis of studies, approximately $10 \%$ of patients with dysplasia were diagnosed on only random biopsies when using chromoendoscopy or high-definition white-light endoscopy and approximately $20 \%$ with standard-definition white-light endoscopy [6, 25, 30, 32, 36-39, 60-67]. Less than $0.2 \%$ of random biopsies were positive for dysplasia [6]. Yet, assuming that endoscopists take at least 30 biopsies per endoscopy (based on the recommended protocol of random 4-quadrant biopsies every $10 \mathrm{~cm}$ for pancolitis [5]), dysplasia would be diagnosed on a random biopsy in approximately 1 in 16 surveillance colonoscopies, a relatively high incidence.

\section{Invisible high-grade dysplasia}

For invisible high-grade dysplasia, current guidelines recommend colectomy given the high risk for synchronous and metachronous carcinoma [5, 13, 23]. Surveillance programs reported rates of synchronous colorectal cancer in 50 to $67 \%$ of colectomy specimens done immediately following the diagnosis of flat high-grade dysplasia [68-71]. The largest study reported synchronous cancer in $16(55 \%)$ of 29 colectomies, while 4 (21\%) of 19 patients with highgrade dysplasia on surveillance progressed to carcinoma after a median follow-up of 2.8 years [68].

\section{Invisible low-grade dysplasia}

The optimal management of invisible low-grade dysplasia, namely, continued surveillance vs. colectomy, is controversial [13]. Previously, invisible low-grade dysplasia was regarded as a high-risk factor for advanced neoplasia. A study from the 1990 s reported that $54 \%$ of cases with lowgrade dysplasia progressed to either high-grade dysplasia or carcinoma [69]. However, the papers on which this was based were not designed to answer that question so these data need to be treated with caution. A meta-analysis in 2007 reported that invisible low-grade dysplasia was associated with concurrent cancer in $22 \%$ of cases [72]. In the remaining cases of low-grade dysplasia, progression to high-grade dysplasia or carcinoma occurred in 14.6\% [72].

More recent studies on invisible low-grade dysplasia have been inconsistent. Some studies reported a low rate of progression to carcinoma [53, 54, 73], while others reported a high rate of progression to high-grade dysplasia or carcinoma [27, 51, 74] as high as 53\% at 5 years [74]. Another issue to consider in the chromoendoscopy era, with visualization of lesions that may have been endoscopically invisible in the past, is whether the results of previous studies on the rates of synchronous or metachronous neoplasia are still applicable today. It also needs to be recalled that carcinomas can arise from low-grade dysplasia (and sometimes less) without ever passing through a phase of high-grade dysplasia [42].

Due to the conflicting results regarding the risk of synchronous or metachronous advanced neoplasia, recommendations for management of invisible low-grade dysplasia have varied. Some authors suggested colectomy for even unifocal disease [27, 74]. The American Society for Gastrointestinal Endoscopy suggested colectomy for multifocal low-grade dysplasia as this indicates widespread dysplasia (reported anecdotally, but for which data are lacking) and an individualized approach for unifocal lowgrade dysplasia [5]. The European Crohn's and Colitis Organization suggested an individualized approach [13]. One has to ask if invisible low-grade dysplasia is to be followed up with surveillance, what is the next node in the management algorithm? Are we waiting for a further biopsy with low-grade dysplasia, high-grade dysplasia or multiple biopsies with low-grade dysplasia before proceeding with colectomy?

\section{Indefinite for dysplasia}

Occasionally, differentiating between dysplasia and regenerative epithelium is difficult. Regenerative changes, especially in the context of inflammation and ulceration, can result in nuclear stratification and crowding, nuclear enlargement, prominent nucleoli, and hyperchromasia that mimic dysplasia. Surface maturation cautions against a diagnosis of dysplasia. However, anecdotal observations, including that from the authors, suggest that surface maturation may occur in cases of so-called "crypt dysplasia", defined as dysplasia limited to crypt epithelium without involvement of the surface. While this is well described in Barrett esophagus, this is a controversial entity in inflammatory bowel disease [45, 75]. Assessment of the surface epithelium may be limited due to an erosion, ulceration, or technical artifacts. In difficult cases, the 
diagnosis of "indefinite for dysplasia" is reasonable, bearing in mind that overtly dysplastic lesions can be superficially eroded [18] and that some cases may be examples of crypt dysplasia. A subset of these "indefinite for dysplasia" cases will show bonafide dysplasia on follow-up [54]. Repeat endoscopy is often done after therapy to reduce the inflammatory background that can obscure pathologic interpretation [5].

\section{Surveillance per the SCENIC guidelines and associated controversies}

Chromoendoscopy was recommended in the SCENIC consensus statement because of its increased detection of dysplastic lesions compared to white-light endoscopy [36, 37]. It was assumed that the increased detection rate and better visualization in conjunction with more thorough removal of dysplasia would lead to a decreased risk of colorectal cancer and mortality. However, concern was expressed about whether chromoendoscopy was detecting clinically relevant lesions that would progress to carcinoma as opposed to more slow-growing and indolent lesions $[76,77]$, but this applies to all forms of dysplasia irrespective of how it is detected. At the time of the SCENIC consensus statement, there was a paucity of data showing a decrease in the incidence of carcinoma and mortality with this modality [76, 77]. Since then, the St. Mark's Hospital surveillance program reported that over a period between 2002 and 2012, those who received chromoendoscopy were less likely to develop cancer than those who did not (2.2 vs. 4.6 cases per 1000 patient-years) [68].

Of particular interest is the potential impact of chromoendoscopy on the rate of metachronous dysplasia or neoplasia, which is detected after the diagnosis of an earlier lesion, and interval carcinoma, defined as cancer that develops despite appropriate surveillance. Interval carcinoma is thought to arise from either a missed lesion on colonoscopy or from a rapidly progressing lesion that develops in between colonoscopies [78]. Interval carcinomas remain a significant problem, as shown in a Dutch study reporting 5 (30\%) interval carcinomas among 17 colorectal cancers arising in the context of inflammatory bowel disease between 2000 and 2014, although only 1\% of patients in its surveillance program developed colorectal cancer [78]. In a study of patients with Crohn's disease who underwent segmental resection or subtotal colectomy for colorectal carcinoma between 2001 and 2011, an alarming $21(85 \%)$ of 25 cases of metachronous colorectal cancer developed while patients were undergoing annual surveillance colonoscopy [79]. This persistent problem of interval and metachronous carcinoma indicates that a significant proportion of carcinomas are not being prevented with endoscopic surveillance. In some patients, like those with a history of colorectal carcinoma in the context of Crohn's disease or invisible dysplasia, colectomy may be preferable to continued surveillance to prevent metachronous carcinomas, even in the era of high-definition endoscopy and chromoendoscopy.

Publications since the SCENIC consensus statement raise caution about the limitations of surveillance colonoscopies even in the era of high-definition endoscopy and chromoendoscopy. Cleveland et al. [80] from the University of Chicago reported that for colectomy specimens resected for dysplasia or neoplasia identified on high-definition endoscopy in patients with inflammatory bowel disease between 2005 and 2014, synchronous invasive carcinoma was present in only $1(3 \%)$ of 36 patients, but $8(22 \%)$ patients had missed synchronous lesions of low-grade dysplasia or high-grade dysplasia. Eluri et al. [81] from Johns Hopkins Hospital looked at resected specimens with high-grade dysplasia or carcinoma from patients with inflammatory bowel disease between 1994 and 2013 and found that in 70 patients 20 had either high-grade dysplasia or invasive carcinoma, which was missed on endoscopy (15 cases of high-grade dysplasia, 5 of carcinoma). Notably, there was no clear trend toward a decline in the proportion of undetected carcinoma or high-grade dysplasia over time, although correlation with high-definition endoscopy or chromoendoscopy could not be precisely determined. The rectum (surprisingly) and the most proximal colon were the sites most likely to contain lesions that escaped detection. As these are both centers that have a longstanding interest in inflammatory bowel disease, these results raise questions on the reliability of endoscopic detection of dysplasia for all patients. In all patients, the risks and benefits of colectomy need to be weighed against the risks and benefits of continued surveillance.

\section{Conclusions}

The SCENIC consensus statement, which has been incorporated into guidelines by the American Society for Gastrointestinal Endoscopy and the European Crohn's and Colitis Organization [5, 13] standardizes terminology for dysplastic lesions in inflammatory bowel disease patients in regard to their endoscopic appearance. The historical implications of associated malignancy and the inconsistency engendered by the DALM terminology should now be avoided. If, however, the term DALM is too ingrained, the term should not be used to equate it with automatic colectomy. A subset of dysplastic lesions may be followed up with continued surveillance instead of immediate surgical resection. Nevertheless, the persistent problems of interval carcinomas and undetected carcinomas and 
dysplasia highlight that endoscopic surveillance may not be feasible for all patients [79-81].

\section{Compliance with ethical standards}

Conflict of interest The authors declare that they have no conflict of interest.

\section{References}

1. Bernstein $\mathrm{CN}$, Blanchard JF, Kliewer E, et al. Cancer risk in patients with inflammatory bowel disease: a population-based study. Cancer. 2001;91:854-62.

2. Crohn B, Rosenberg H. The sigmoidoscopic picture of chronic ulcerative colitis (non-specific). Am J Med Sci. 1925;170:220-8.

3. Lockhart-Mummery P. The causes of colitis, with special reference to its surgical treatment with an account of 36 cases. Med Chir Trans. 1907;90:589-618.

4. Farraye FA, Odze RD, Eaden J, et al. AGA medical position statement on the diagnosis and management of colorectal neoplasia in inflammatory bowel disease. Gastroenterology. 2010;138:738-45.

5. American Society for Gastrointestinal Endoscopy Standards of Practice Committee, Shergill AK, Lightdale JR, et al. The role of endoscopy in inflammatory bowel disease. Gastrointest Endosc. 2015;81:1101-21 e1-13.

6. Laine L, Kaltenbach T, Barkun A, et al. SCENIC international consensus statement on surveillance and management of dysplasia in inflammatory bowel disease. Gastroenterology. 2015;148: 639-51 e28.

7. Blackstone MO, Riddell RH, Rogers $\mathrm{BH}$, et al. Dysplasiaassociated lesion or mass (DALM) detected by colonoscopy in long-standing ulcerative colitis: an indication for colectomy. Gastroenterology. 1981;80:366-74.

8. Bernstein CN, Shanahan F, Weinstein WM. Are we telling patients the truth about surveillance colonoscopy in ulcerative colitis? Lancet. 1994;343:71-4.

9. Torres C, Antonioli D, Odze RD. Polypoid dysplasia and adenomas in inflammatory bowel disease: a clinical, pathologic, and follow-up study of 89 polyps from 59 patients. Am J Surg Pathol. 1998;22:275-84.

10. Engelsgjerd M, Farraye FA, Odze RD. Polypectomy may be adequate treatment for adenoma-like dysplastic lesions in chronic ulcerative colitis. Gastroenterology. 1999;117:1288-94.

11. Odze RD, Farraye FA, Hecht JL, et al. Long-term follow-up after polypectomy treatment for adenoma-like dysplastic lesions in ulcerative colitis. Clin Gastroenterol Hepatol. 2004;2:534-41.

12. Rubin PH, Friedman S, Harpaz N, et al. Colonoscopic polypectomy in chronic colitis: conservative management after endoscopic resection of dysplastic polyps. Gastroenterology. 1999;117:1295-300.

13. Magro F, Gionchetti P, Eliakim R, et al. Third European evidencebased consensus on diagnosis and management of ulcerative colitis. Part 1: definitions, diagnosis, extra-intestinal manifestations, pregnancy, cancer surveillance, surgery, and ileo-anal pouch disorders. J Crohns Colitis. 2017;11:649-70.

14. Rutter MD, Saunders BP, Wilkinson KH, et al. Most dysplasia in ulcerative colitis is visible at colonoscopy. Gastrointest Endosc. 2004;60:334-9.

15. Tytgat GN, Dhir V, Gopinath N. Endoscopic appearance of dysplasia and cancer in inflammatory bowel disease. Eur J Cancer. 1995;31A:1174-7.
16. Butt JH, Konishi F, Morson BC, et al. Macroscopic lesions in dysplasia and carcinoma complicating ulcerative colitis. Dig Dis Sci. 1983;28:18-26.

17. Rosenstock E, Farmer RG, Petras R, et al. Surveillance for colonic carcinoma in ulcerative colitis. Gastroenterology. 1985;89:1342-6.

18. Riddell RH, Goldman H, Ransohoff DF, et al. Dysplasia in inflammatory bowel disease: standardized classification with provisional clinical applications. Hum Pathol. 1983;14:931-68.

19. Odze RD. Adenomas and adenoma-like DALMs in chronic ulcerative colitis: a clinical, pathological, and molecular review. Am J Gastroenterol. 1999;94:1746-50.

20. Mueller E, Vieth M, Stolte M, et al. The differentiation of true adenomas from colitis-associated dysplasia in ulcerative colitis: a comparative immunohistochemical study. Hum Pathol. 1999;30: 898-905.

21. Wanders LK, Dekker E, Pullens B, et al. Cancer risk after resection of polypoid dysplasia in patients with longstanding ulcerative colitis: a meta-analysis. Clin Gastroenterol Hepatol. 2014;12:756-64.

22. Farraye FA, Odze RD, Eaden J, et al. AGA technical review on the diagnosis and management of colorectal neoplasia in inflammatory bowel disease. Gastroenterology. 2010;138:746-74. 74 e1-4; quize12-3.

23. Annese V, Daperno M, Rutter MD, et al. European evidence based consensus for endoscopy in inflammatory bowel disease. $\mathrm{J}$ Crohns Colitis. 2013;7:982-1018.

24. Soetikno R, Subramanian V, Kaltenbach T, et al. The detection of nonpolypoid (flat and depressed) colorectal neoplasms in patients with inflammatory bowel disease. Gastroenterology. 2013;144:1349-52. 52 e1-6.

25. Blonski W, Kundu R, Lewis J, et al. Is dysplasia visible during surveillance colonoscopy in patients with ulcerative colitis? Scand J Gastroenterol. 2008;43:698-703.

26. Rubin DT, Rothe JA, Hetzel JT, et al. Are dysplasia and colorectal cancer endoscopically visible in patients with ulcerative colitis? Gastrointest Endosc. 2007;65:998-1004.

27. Choi CH, Ignjatovic-Wilson A, Askari A, et al. Low-grade dysplasia in ulcerative colitis: risk factors for developing high-grade dysplasia or colorectal cancer. Am J Gastroenterol. 2015;110:1461-71. quiz 72.

28. Velayos F, Kathpalia P, Finlayson E. Changing paradigms in detection of dysplasia and management of patients with inflammatory bowel disease: is colectomy still necessary? Gastroenterology. 2017;152:440-50 e1.

29. Subramanian V, Ramappa V, Telakis E, et al. Comparison of high definition with standard white light endoscopy for detection of dysplastic lesions during surveillance colonoscopy in patients with colonic inflammatory bowel disease. Inflamm Bowel Dis. 2013;19:350-5.

30. Rutter MD, Saunders BP, Schofield G, et al. Pancolonic indigo carmine dye spraying for the detection of dysplasia in ulcerative colitis. Gut. 2004;53:256-60.

31. Matsumoto T, Nakamura S, Jo Y, et al. Chromoscopy might improve diagnostic accuracy in cancer surveillance for ulcerative colitis. Am J Gastroenterol. 2003;98:1827-33.

32. Marion JF, Waye JD, Present DH, et al. Chromoendoscopytargeted biopsies are superior to standard colonoscopic surveillance for detecting dysplasia in inflammatory bowel disease patients: a prospective endoscopic trial. Am J Gastroenterol. 2008;103:2342-9.

33. Carballal S, Maisterra S, Lopez-Serrano A, et al. Real-life chromoendoscopy for neoplasia detection and characterisation in longstanding IBD. Gut. 2018;67:70-8.

34. Deepak P, Hanson GJ, Fletcher JG, et al. Incremental diagnostic yield of chromoendoscopy and outcomes in inflammatory bowel 
disease patients with a history of colorectal dysplasia on whitelight endoscopy. Gastrointest Endosc. 2016;83:1005-12.

35. Marion JF, Waye JD, Israel Y, et al. Chromoendoscopy is more effective than standard colonoscopy in detecting dysplasia during long-term surveillance of patients with colitis. Clin Gastroenterol Hepatol. 2016;14:713-9.

36. Kiesslich R, Goetz M, Lammersdorf K, et al. Chromoscopyguided endomicroscopy increases the diagnostic yield of intraepithelial neoplasia in ulcerative colitis. Gastroenterology. 2007;132:874-82.

37. Kiesslich R, Fritsch J, Holtmann M, et al. Methylene blue-aided chromoendoscopy for the detection of intraepithelial neoplasia and colon cancer in ulcerative colitis. Gastroenterology. 2003;124:880-8.

38. Gunther U, Kusch D, Heller F, et al. Surveillance colonoscopy in patients with inflammatory bowel disease: comparison of random biopsy vs. targeted biopsy protocols. Int J Colorectal Dis. 2011;26:667-72.

39. Hlavaty T, Huorka M, Koller T, et al. Colorectal cancer screening in patients with ulcerative and Crohn's colitis with use of colonoscopy, chromoendoscopy and confocal endomicroscopy. Eur J Gastroenterol Hepatol. 2011;23:680-9.

40. Leong RW, Ooi M, Corte C, et al. Full-spectrum endoscopy improves surveillance for dysplasia in patients with inflammatory bowel diseases. Gastroenterology. 2017;152:1337-44 e3.

41. Workshop PitP. The Paris endoscopic classification of superficial neoplastic lesions: esophagus, stomach, and colon. Gastrointest Endosc. 2003;58:S3-43.

42. Levi GS, Harpaz N. Intestinal low-grade tubuloglandular adenocarcinoma in inflammatory bowel disease. Am J Surg Pathol. 2006;30:1022-9.

43. Schlemper RJ, Riddell RH, Kato Y, et al. The Vienna classification of gastrointestinal epithelial neoplasia. Gut. 2000;47:251-5.

44. Gordon IO. Inflammatory bowel disease. In: Noffsinger A, editor. Fenoglio-Preiser's gastrointestinal pathology. 4th ed. Philadelphia, PA: Lippincott Williams \& Wilkins; 2017. pp. 547-643.

45. Patil DT, Greenson JK, Odze RD. Inflammatory disorders of the large intestine. In: Odze RD, Goldblum JR, editors. Odze and Goldblum surgical pathology of the GI tract, liver, biliary tract and pancreas. 3rd ed. Philadelphia, PA: Elsevier Inc.; 2015. pp. 436-511.

46. Geboes K, Riddell R, Jain D. Inflammatory bowel diseases. In: Riddell R, Jain D, editors. Lewin, Weinstein, and Riddell's gastrointestinal pathology and its clinical implications volume 2. 2nd ed. Philadelphia, PA: Lippincott Williams \& Wilkins; 2014. pp. 983-1208.

47. Riddell RH. Premalignant and early malignant lesions in the gastrointestinal tract: definitions, terminology, and problems. Am J Gastroenterol. 1996;91:864-72.

48. Odze RD. Pathology of dysplasia and cancer in inflammatory bowel disease. Gastroenterol Clin North Am. 2006;35:533-52.

49. Itzkowitz SH, Harpaz N. Diagnosis and management of dysplasia in patients with inflammatory bowel diseases. Gastroenterology. 2004;126:1634-48.

50. Ten Hove JR, Mooiweer E, Dekker E, et al. Low rate of dysplasia detection in mucosa surrounding dysplastic lesions in patients undergoing surveillance for inflammatory bowel diseases. Clin Gastroenterol Hepatol. 2017;15:222-8 e2.

51. Goldstone R, Itzkowitz S, Harpaz N, et al. Progression of lowgrade dysplasia in ulcerative colitis: effect of colonic location. Gastrointest Endosc. 2011;74:1087-93.

52. Kisiel JB, Loftus EV Jr., Harmsen WS, et al. Outcome of sporadic adenomas and adenoma-like dysplasia in patients with ulcerative colitis undergoing polypectomy. Inflamm Bowel Dis. 2012;18:226-35.
53. Navaneethan U, Jegadeesan R, Gutierrez NG, et al. Progression of low-grade dysplasia to advanced neoplasia based on the location and morphology of dysplasia in ulcerative colitis patients with extensive colitis under colonoscopic surveillance. J Crohns Colitis. 2013; 7:e684-91.

54. Pekow JR, Hetzel JT, Rothe JA, et al. Outcome after surveillance of low-grade and indefinite dysplasia in patients with ulcerative colitis. Inflamm Bowel Dis. 2010;16:1352-6.

55. van Schaik FD, Mooiweer E, van der Have M, et al. Adenomas in patients with inflammatory bowel disease are associated with an increased risk of advanced neoplasia. Inflamm Bowel Dis. 2013;19:342-9.

56. East JE, Toyonaga T, Suzuki N. Endoscopic management of nonpolypoid colorectal lesions in colonic IBD. Gastrointest Endosc Clin N Am. 2014;24:435-45.

57. Dixon MF, Brown LJ, Gilmour HM, et al. Observer variation in the assessment of dysplasia in ulcerative colitis. Histopathology. 1988;13:385-97.

58. Eaden J, Abrams K, McKay H, et al. Inter-observer variation between general and specialist gastrointestinal pathologists when grading dysplasia in ulcerative colitis. J Pathol. 2001;194:152-7.

59. Odze RD, Goldblum J, Noffsinger A, et al. Interobserver variability in the diagnosis of ulcerative colitis-associated dysplasia by telepathology. Mod Pathol. 2002;15:379-86.

60. Dekker E, van den Broek FJ, Reitsma JB, et al. Narrow-band imaging compared with conventional colonoscopy for the detection of dysplasia in patients with longstanding ulcerative colitis. Endoscopy. 2007;39:216-21.

61. Efthymiou M, Allen PB, Taylor AC, et al. Chromoendoscopy versus narrow band imaging for colonic surveillance in inflammatory bowel disease. Inflamm Bowel Dis. 2013;19:2132-8.

62. Ignjatovic A, East JE, Subramanian V, et al. Narrow band imaging for detection of dysplasia in colitis: a randomized controlled trial. Am J Gastroenterol. 2012;107:885-90.

63. Jaramillo E, Watanabe M, Befrits R, et al. Small, flat colorectal neoplasias in long-standing ulcerative colitis detected by highresolution electronic video endoscopy. Gastrointest Endosc. 1996;44:15-22.

64. Picco MF, Pasha S, Leighton JA, et al. Procedure time and the determination of polypoid abnormalities with experience: implementation of a chromoendoscopy program for surveillance colonoscopy for ulcerative colitis. Inflamm Bowel Dis. 2013;19:1913-20.

65. van den Broek FJ, Fockens P, van Eeden S, et al. Narrow-band imaging versus high-definition endoscopy for the diagnosis of neoplasia in ulcerative colitis. Endoscopy. 2011;43:108-15.

66. van den Broek FJ, Fockens P, van Eeden S, et al. Endoscopic trimodal imaging for surveillance in ulcerative colitis: randomised comparison of high-resolution endoscopy and autofluorescence imaging for neoplasia detection; and evaluation of narrow-band imaging for classification of lesions. Gut. 2008;57:1083-9.

67. van den Broek FJ, Stokkers PC, Reitsma JB, et al. Random biopsies taken during colonoscopic surveillance of patients with longstanding ulcerative colitis: low yield and absence of clinical consequences. Am J Gastroenterol. 2014;109:715-22.

68. Choi CH, Rutter MD, Askari A, et al. Forty-year analysis of colonoscopic surveillance program for neoplasia in ulcerative colitis: an updated overview. Am J Gastroenterol. 2015;110: 1022-34.

69. Connell WR, Lennard-Jones JE, Williams CB, et al. Factors affecting the outcome of endoscopic surveillance for cancer in ulcerative colitis. Gastroenterology. 1994;107:934-44.

70. Friedman S, Rubin PH, Bodian C, et al. Screening and surveillance colonoscopy in chronic Crohn's colitis. Gastroenterology. 2001;120:820-6. 
71. Hata K, Watanabe T, Kazama S, et al. Earlier surveillance colonoscopy programme improves survival in patients with ulcerative colitis associated colorectal cancer: results of a 23-year surveillance programme in the Japanese population. $\mathrm{Br} \mathrm{J}$ Cancer. 2003;89:1232-6.

72. Thomas T, Abrams KA, Robinson RJ, et al. Meta-analysis: cancer risk of low-grade dysplasia in chronic ulcerative colitis. Aliment Pharmacol Ther. 2007;25:657-68.

73. Fumery M, Dulai PS, Gupta S, et al. Incidence, risk factors, and outcomes of colorectal cancer in patients with ulcerative colitis with low-grade dysplasia: a systematic review and meta-analysis. Clin Gastroenterol Hepatol. 2017;15:665-74 e5.

74. Ullman T, Croog V, Harpaz N, et al. Progression of flat low-grade dysplasia to advanced neoplasia in patients with ulcerative colitis. Gastroenterology. 2003;125:1311-9.

75. Odze RD, Maley CC. Neoplasia without dysplasia: lessons from Barrett esophagus and other tubal gut neoplasms. Arch Pathol Lab Med. 2010;134:896-906.

76. Higgins PD. Miles to go on the SCENIC route: should chromoendoscopy become the standard of care in IBD surveillance? Am J Gastroenterol. 2015;110:1035-7.
77. Marion JF, Sands BE. The SCENIC consensus statement on surveillance and management of dysplasia in inflammatory bowel disease: praise and words of caution. Gastroenterology. 2015;148:462-7.

78. Mooiweer E, van der Meulen-de Jong AE, Ponsioen CY, et al. Incidence of interval colorectal cancer among inflammatory bowel disease patients undergoing regular colonoscopic surveillance. Clin Gastroenterol Hepatol. 2015;13:1656-61.

79. Maser EA, Sachar DB, Kruse D, et al. High rates of metachronous colon cancer or dysplasia after segmental resection or subtotal colectomy in Crohn's colitis. Inflamm Bowel Dis. 2013;19: $1827-32$.

80. Cleveland NK, Colman RJ, Rodriquez D, et al. Surveillance of IBD using high definition colonoscopes does not miss adenocarcinoma in patients with low-grade dysplasia. Inflamm Bowel Dis. 2016;22:631-7.

81. Eluri S, Parian AM, Limketkai BN, et al. Nearly a third of high-grade dysplasia and colorectal cancer is undetected in patients with inflammatory bowel disease. Dig Dis Sci. 2017;62: 3586-93. 